\title{
A genetic link between cold responses and flowering time through FVE in Arabidopsis thaliana
}

\author{
Hyoun-Joung Kim ${ }^{1,5}$, Youbong Hyun ${ }^{2,5}$, Jin-Young Park ${ }^{1,3,5}$, Mi-Jin Park ${ }^{1}$, Mi-Kyung Park ${ }^{1}$, Myoung Duck Kim ${ }^{1}$, \\ Hye-Joung Kim ${ }^{1}$, Mi Hyun Lee ${ }^{1}$, Jihyun Moon ${ }^{2}$, Ilha Lee ${ }^{2,4} \&$ Jungmook Kim ${ }^{1,3}$
}

Cold induces expression of a number of genes that encode proteins that enhance tolerance to freezing temperatures in plants ${ }^{1,2}$. A cis-acting element responsive to cold and drought, the C-repeat/dehydration-responsive element (C/DRE), was identified in the Arabidopsis thaliana stress-inducible genes $R D 29 A^{3}$ and $C O R 15 a^{4}$ and found in other cold-inducible genes in various plants ${ }^{1,5}$. C/DRE-binding factor/DRE-binding protein (CBF/DREB) is an essential component of the cold-acclimation response $^{1,2}$, but the signaling pathways and networks are mostly unknown. Here we used targeted genetic approach to isolate A. thaliana mutants with altered cold-responsive gene expression (acg) and identify ACG1 as a negative regulator of the CBF/DREB pathway. acg1 flowered late and had elevated expression of FLOWERING LOCUS C $(F L C)^{6}$, a repressor of flowering encoding a MADS-box protein. We showed that acg1 is a null allele of the autonomous pathway gene FVE. FVE encodes a homolog of the mammalian retinoblastomaassociated protein, a component of a histone deacetylase (HDAC) complex involved in transcriptional repression ${ }^{7,8}$. We also showed that plants sense intermittent cold stress through FVE and delay flowering with increasing expression of $F L C$. Dual roles of FVE in regulating the flowering time and the cold response may have an evolutionary advantage for plants by increasing their survival rates. a

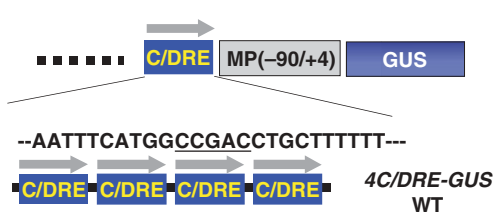

b
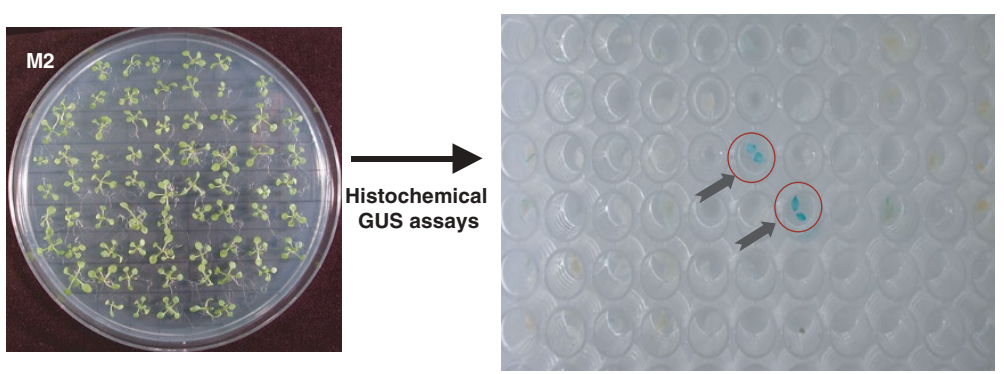

d

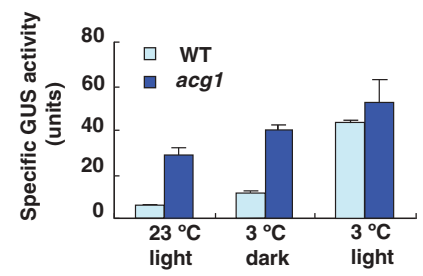

Figure 1 Isolation and genetic analysis of acg1 mutants with constitutive GUS expression at $23^{\circ} \mathrm{C}$ and the late-flowering phenotype. (a) Diagram of the chimeric construct used for generating 4C/DRE-GUS transgenic $A$. thaliana. WT, wild-type. (b) A typical example of screening that shows putative 4C/DREGUS mutants with constitutive GUS expression. (c) Genetic analysis of acg1 mutants by backcrossing with wild-type plants. ND, not determined.

(d) Fluorometric quantification of GUS activities of acg1 mutants and wild-type plants (WT). Specific activities (units) are shown as pmol reaction product (4-methylumbelliferone) generated per min per mg of total protein. Data are mean \pm standard error of three independent treatments.

${ }^{1}$ Kumho Life and Environmental Science Laboratory, 1 Oryong-Dong, Puk-Gu, Gwangju 500-712, Korea. ${ }^{2}$ School of Biological Sciences, Seoul National University, Seoul 151-742, Korea. ${ }^{3}$ Agricultural Plant Stress Research Center (APSRC), Chonnam National University, Gwangju 500-757, Korea. ${ }^{4}$ Plant Metabolism Research Center, Kyung Hee University, Suwon 449-701, Korea. ${ }^{5}$ These authors contributed equally to this work. Correspondence should be addressed to J.K.

(jungmookkim@hanmail.net).

Published online 25 January 2004; doi:10.1038/ng1298 
To circumvent the problems associated with the complicated interactive effects of various cis-acting elements in the whole promoter region in studying signaling pathways and networks, we generated transgenic A. thaliana with four copies of the synthetic C/DRE derived from the COR 15 a promoter, fused to a GUS reporter gene with a minimal promoter containing a TATA box (4C/DRE-GUS), that responds to cold but not to other stress conditions, such as abscisic acid or high salinity (Fig. 1a) ${ }^{9}$. Light signaling mediated by phytochrome $\mathrm{B}$ is necessary for cold-induced gene expression through the C/DRE 9 . We mutagenized 4C/DRE-GUS plants by ethylmethane sulfonate and screened the resulting M2 population for mutants with constitutive GUS expression at $23{ }^{\circ} \mathrm{C}$ or under cold

\section{a}

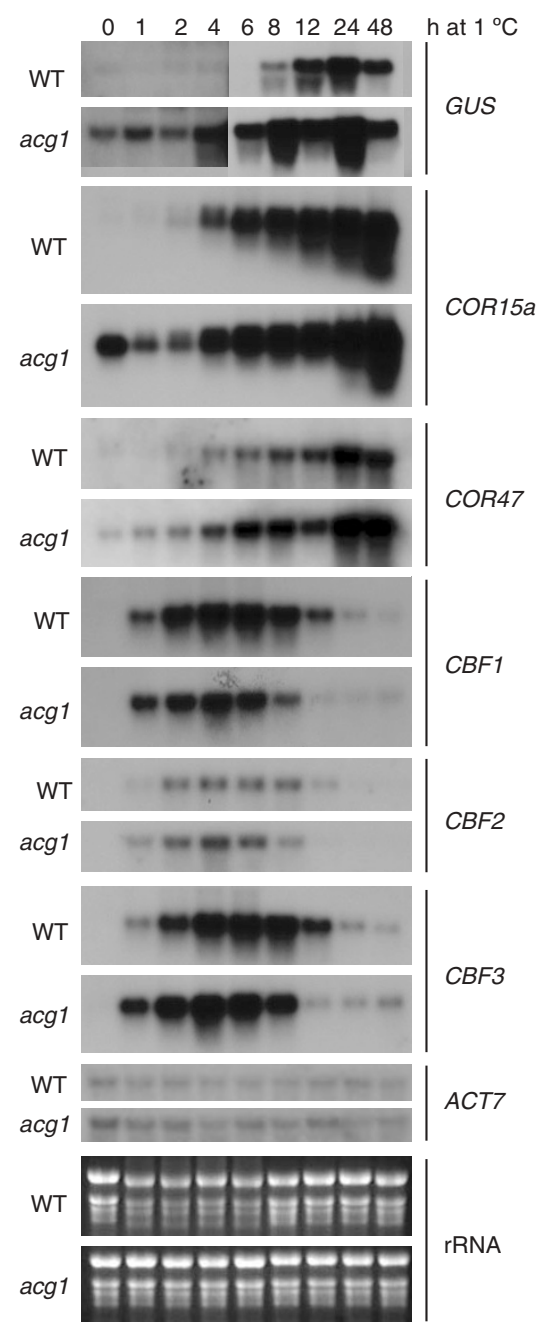

b

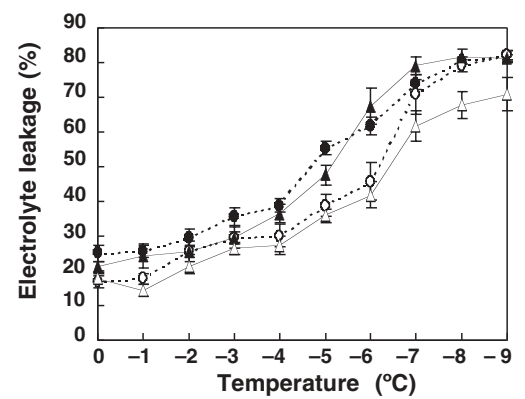

stress but without light by carrying out histochemical GUS assays on two excised leaves (Fig. 1b). We observed dwarfism and growth retardation in most mutants (data not shown) and delay in flowering in some mutants. We studied the acg1 mutant, which had the most severe delay in flowering, to explore the genetic link between coldstress signaling and the phase transition to flowering. Genetic analysis of the acg1 mutant showed that aberrant GUS expression at $23^{\circ} \mathrm{C}$ was due to a single recessive nuclear mutation that cosegregated with the late-flowering phenotype (Fig. 1c). acgl mutants had higher GUS expression at $23{ }^{\circ} \mathrm{C}$ or $3{ }^{\circ} \mathrm{C}$ in the dark than $4 C / D R E-G U S$ plants (hereafter called wild-type; Fig. 1d). Cold treatment in the light further enhanced GUS expression in acg1 relative to wild-type plants.

To investigate alteration of cold-responsive gene expression in acg1 mutants, we examined the time course of GUS induction in response to cold in acg1 and wild-type plants using northern-blot analysis (Fig. 2a). The level of GUS mRNA was higher in acg1 mutants than in wildtype plants before cold treatment and began in increase in acg1 mutants, but not wild-type plants, $4 \mathrm{~h}$ after cold treatment. By 6-8 h after cold treatment, the level of GUS mRNA was much higher in mutants than in wild-type plants. Longer cold treatment substantially increased GUS mRNA levels in mutants and wild-type plants to similar levels.

To test whether the mutation affected expression of $C O R$ genes containing the C/DRE, we analyzed induction kinetics of COR $15 a$ and COR47 and observed an expression pattern similar to that of GUS, although expression of COR 47 was not as robust as that of COR15a. We could not detect the transcripts of three $C B F$ genes at $23^{\circ} \mathrm{C}$ in acgl, suggesting that the constitutive expression of GUS and COR in acgl at normal temperatures was not caused by enhanced expression of $C B F$. We observed similarly increased levels of $C B F$ transcripts in response to cold in wild-type plants and mutants, but the transient increase in $C B F$ expression occurred earlier in acgl mutants than in wild-type plants. Thus, enhanced expression of COR15a in response to cold may be due, in part, to earlier expression of $C B F$. These results indicate that the acg1 mutation impairs the repression of the CBF/DREB pathway.

To test whether the acg1 mutation had an effect on freezing tolerance, we conducted an electrolyte leakage test ${ }^{10}$ on 2-week-old acg1 mutant and wild-type plants before and after preincubation at $4{ }^{\circ} \mathrm{C}$ for $2 \mathrm{~d}$ (Fig. 2b). Nonacclimated acg1 and wild-type plants showed similar tolerance to freezing, but when cold-acclimated, acgl mutants had greater freezing tolerance than wild-type plants, in the range of -7 to $-9{ }^{\circ} \mathrm{C}$. This difference is statistically significant and may be due to the enhanced upregulation of $C O R$ genes in response to cold.

acg1 mutants flowered later than wild-type plants under both long days and short days, and the flowering time of acg1 mutants was reduced to wild-type level by vernalization treatment (Fig. 3a-c), suggestive of a mutation in the autonomous pathway. In A. thaliana,

Figure 2 Cold-responsive gene expression and electrolyte leakage of acg1 mutants and wild-type plants. (a) Northern-blot analyses of cold-responsive gene expression in acg1 mutants and wild-type plants (WT). Plants were incubated at $1{ }^{\circ} \mathrm{C}$ with varying times in the light. The X-ray film exposure time of northern blots of the GUS mRNA from the samples treated for 6-48 h was three times shorter than that of the samples treated for 1-4 h. Actin 7 (ACT7) mRNA was used as a loading control for RNA samples. (b) Electrolyte leakage of leaves from acg1 mutants and wild-type plants at different temperatures with or without cold acclimation (incubation at $1{ }^{\circ} \mathrm{C}$ for $2 \mathrm{~d}$ in the light). Data are mean \pm standard error of at least 10 different samples. Broken lines, wild-type plants; solid lines, acg1 mutants; filled circles, wildtype plants without cold acclimation; filled triangles, acg1 mutants without cold acclimation; open circles, wild-type plants with cold acclimation; open triangles, acg1 mutants with cold acclimation. 
Figure 3 Analysis of the flowering time and expression of floral pathway genes and COR15a in acg1 and other late-flowering mutants. (a) 6-weekold wild-type (WT; left) and acg1 (right) plants grown under long-day conditions ( $16 \mathrm{~h}$ light- $8 \mathrm{~h}$ dark). (b) The rosette leaf numbers of acg 1 mutants and wild-type plants (WT) vernalized for 6 weeks (solid column) or not vernalized (open column) and grown under long-day conditions. Time to flowering was measured as the number of rosette leaves formed on the main shoot. (c) The rosette leaf numbers of acg1 mutants and wildtype plants (WT) grown under short-day conditions ( $8 \mathrm{~h}$ light-16 h dark). Counting of the rosette leaf number of acg1 mutants was stopped after growing the plants for 7 months. (d) RT-PCR analysis of expression of $F L C, S O C 1$ and $F T$ in Col-0, acg1 and other late-flowering mutants. $\beta$-tubulin (TUBB) mRNA was used as a loading control for RNA samples. (e) Northern-blot analysis of COR15a expression in fve-3, fve-4, fve- $4 \mathrm{flc}-3$ and $\mathrm{flc}-3$ mutants compared with acg1 and Col-0 plants. a

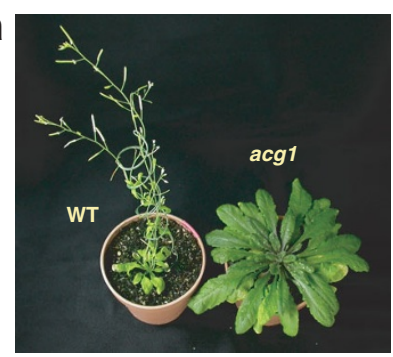

b

d

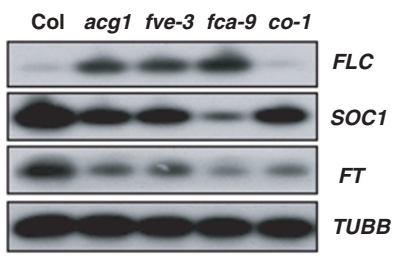

C

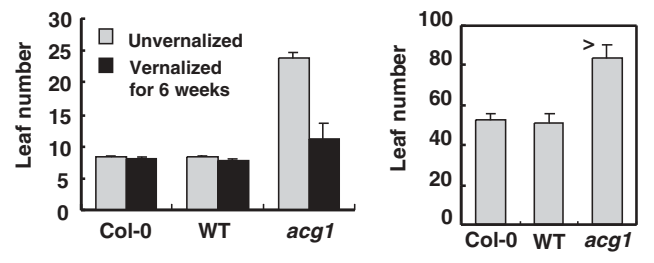

e

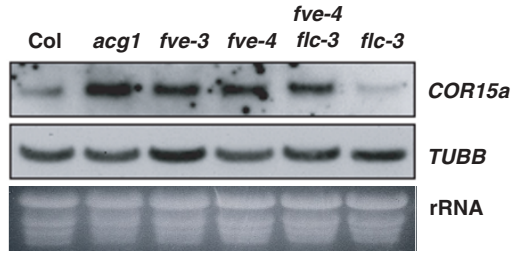

mutations in autonomous pathway genes cause elevated FLC expression and late flowering, but vernalization offsets this effect ${ }^{11}$. FLC expression was higher in acg1 mutants than in Columbia-0 (Col-0) plants, similar to the level of expression seen in fve-3 plants (Fig. 3d). fca-9 (ref. 12) plants, which flowered later than acg1 and fve-3 (ref. 13) plants, had a higher FLC level, but $c o-1$ (ref. 14) plants, which are lateflowering only in long-day photoperiods, had a similar FLC level as Col-0. FLC negatively regulates the expression of SUPPRESSOR OF OVEREXPRESSION OF CO 1 (SOC1), encoding a MADS-box transcription factor, and FLOWERING LOCUS T (FT), encoding a protein with similarity to a Raf kinase inhibitor, both of which activate flower meristem identity genes ${ }^{15}$. We observed decreased levels of SOC1 and FT in acgl mutants as well as in $f v e-3$ and $f c a-9$ plants. These results and complementation tests (Supplementary Fig. 1 online) suggest that late flowering in acg1 mutants is due to elevated FLC expression and that acg1 is an allele of $f v e$, an autonomous pathway gene ${ }^{13,16}$. Expression of COR $15 \mathrm{a}$ at $23^{\circ} \mathrm{C}$ was enhanced in $f v e-3$, fve-4, fve-4 flc3 plants, as in acg1 mutants, but expression in $f l c-3$ plants (Fig. 3e) was similar to that seen in Col-0 plants, suggesting that the effect of $F V E$ on expression of cold-responsive genes is independent of FLC function.

$F V E$ was isolated by positional cloning ${ }^{17}$. Linkage analysis confirmed that acg1 might be fve, an allele of FVE (Fig. 4a). acg1 or fve-3 has a point mutation that converts Trp46 or Trp142, respectively, into a premature stop codon (Fig. $\mathbf{4 b}$ ). The level of FVE mRNA was much lower in acg1 mutants than in Col-0 and wild-type plants, suggestive of mRNA instability due to a premature termination (Fig. 4c). The protein encoded by FVE shows high sequence homology to the mammalian retinoblastoma-associated protein (RbAp; Supplementary Fig. 2 online) and is a

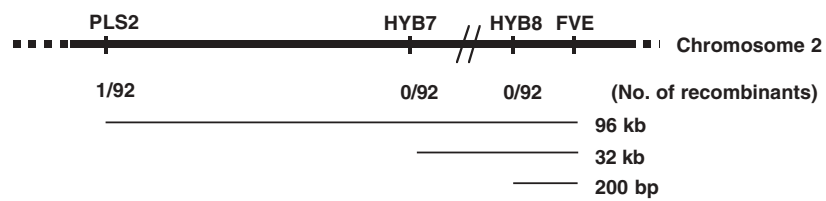

b

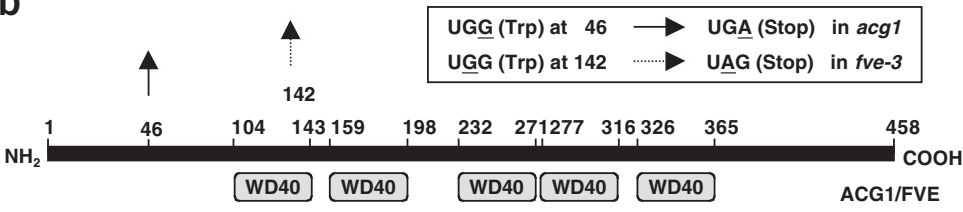

Figure 4 acg1 is a null allele of FVE that encodes a plant homolog of the mammalian RbAp. (a) Linkage analysis of the late-flowering phenotype of acg1 with FVE. Homozygous acg1 mutants in the segregated $\mathrm{F}_{2}$ population obtained from crossing acg1 with the ecotype Landsberg erecta were used for mapping. (b) Scheme of domain structures of the protein encoded by FVE and positions of nonsense mutations in acg1 and fve-3. Numbers above the thick column denote the amino acid numbers. WD40 indicates WD-40-repeat domain predicted by a Motif Scan program 25 . (c) Northern blot showing the FVE mRNA level in Col-0, wild-type (WT) and acg1 plants. (d) Northern-blot analysis of FVE expression in response to cold in Col-O plants. Plants were incubated at $1{ }^{\circ} \mathrm{C}$ for varying periods of time (h). COR15a was analyzed for monitoring cold-inducible gene expression.
C

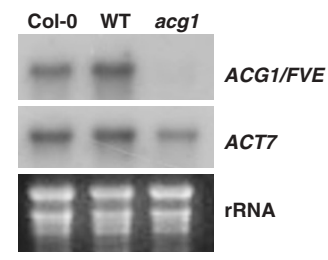

d

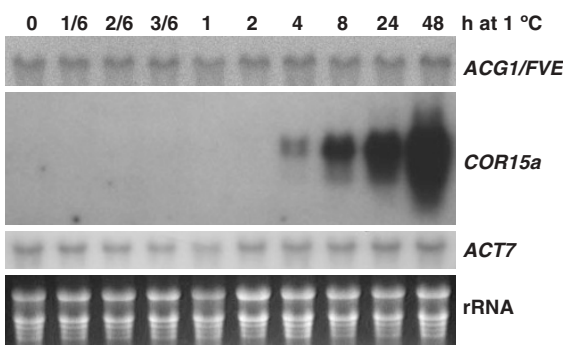


a

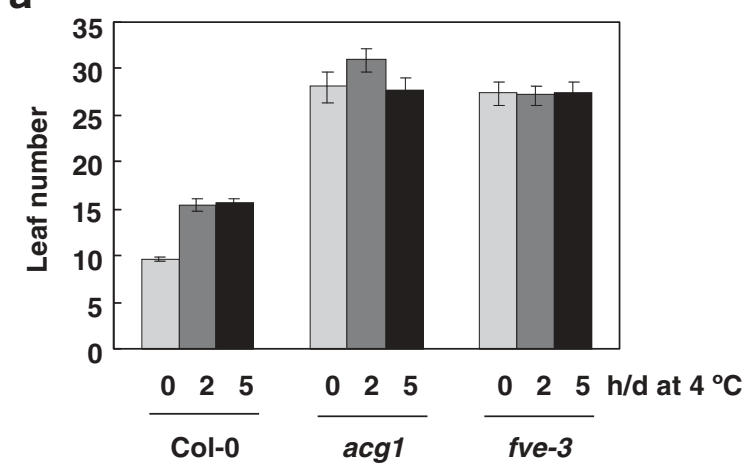

b

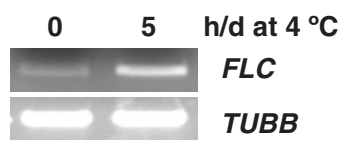

C

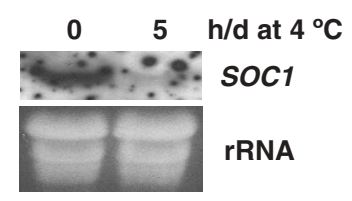

Figure 5 Intermittent cold treatment delays the flowering of wild-type plants but not that of acgl and fve mutants. (a) Plants were incubated at $4{ }^{\circ} \mathrm{C}$ for $2 \mathrm{~h}$ or $5 \mathrm{~h}$ every day, and the rosette leaf numbers were measured at bolting. RT-PCR analysis of FLC expression (b) and northern-blot analysis of $\mathrm{SOCl}$ expression (c) in Col-0 plants subjected to intermittent cold-stress treatment.

predicted to contain five WD-40 repeats, domains that are found in multiprotein complexes involved in numerous cellular processes ${ }^{18}$. In mammals, the retinoblastoma $(\mathrm{Rb})$ protein represses transcription by recruiting HDAC involved in repressing gene expression by deacetylation of histone residues ${ }^{7}$. RbAp interacts with HDAC associated with $\mathrm{Rb}$ and has a role in transcriptional repression ${ }^{8}$. These mechanisms seem to be conserved in plants, although the biological roles of the homologous proteins are not known ${ }^{19,20}$. A loss-of-function mutation in FVE could release the transcriptional repression mediated by HDAC, resulting in aberrant expression of GUS and COR at normal temperatures. This alteration of $C O R$ expression could affect a negative feedback loop of $C B F$ expression, shifting it to an earlier time. Release of the transcriptional repression and earlier $C B F$ expression could enhance the response of COR expression to cold in acg1 mutants (Fig. 2a). FVE mRNA was not affected by cold (Fig. 4d).

Because the same mutation caused both enhanced response of coldinduced gene expression and late flowering, we tested whether cold stress affected flowering time by treating plants at $4{ }^{\circ} \mathrm{C}$ for $2 \mathrm{~h}$ or $5 \mathrm{~h}$ every day during the entire growth period. Intermittent cold treatment substantially delayed flowering in wild-type plants but had no effect on acgl and fve mutants (Fig. 5a). Moreover, intermittent cold treatment increased the level of $F L C$ (Fig. $5 \mathbf{b}$ ) and thereby decreased SOC1 expression (Fig. 5c). Genetic and expression analyses of various flowering-time mutants have indicated that a low ambient growth temperature (such as $16^{\circ} \mathrm{C}$ ) is sensed through a genetic pathway requiring both $F C A$ and $F V E^{21}$. Our results suggest that plants sense intermittent cold stress through FVE and delay flowering time by increasing the level of FLC. Consistent with a proposed role of FVE as a component of HDAC, the fve mutant had an increase in acetylation of $F L C$, as shown by chromatin immunoprecipitation analysis of the acetylation state of histone $\mathrm{H} 4$ at $F L C^{22}$. Dual roles of FVE in regulating the flowering time and the stress response to cold may provide evolutionary fitness to plants, particularly in enduring the cold spell of early spring.

\section{METHODS}

Plant materials and growth conditions. We used the ecotype Columbia- 0 for all experiments. We obtained the late-flowering mutants $f v e-3, f c a-9, l d-1$ and $c o-1$ from Arabidopsis Biological Resource Center and $f l d-3, f v e-4$, fve- $4 f c-3$ and $f l c-3$ seeds from R. Amasino (University of Wisconsin, Madison). We grew plants for $10 \mathrm{~d}$ on agar medium under long-day conditions $(16 \mathrm{~h}$ light- $8 \mathrm{~h}$ dark $)$ at $23^{\circ} \mathrm{C}$ and used them for cold treatment at $1-4{ }^{\circ} \mathrm{C}$ or for expression analysis of floral pathway genes. For flowering time analysis, we grew plants on soil under longday ( $16 \mathrm{~h}$ light- $8 \mathrm{~h}$ dark) or short-day conditions ( $8 \mathrm{~h}$ light- $-16 \mathrm{~h}$ dark). For analysis of the effect of intermittent cold stress on the flowering time, we incubated plants on soil every day at $4{ }^{\circ} \mathrm{C}$ for 0,2 or $5 \mathrm{~h}$, starting $4 \mathrm{~h}$ after dawn.

Mutagenesis, mutant isolation and genetic analysis. We mutagenized 4C/DREGUS seeds (approximately 20,000 seeds) with ethylmethane sulfonate and harvested M2 seeds. We planted M2 seeds individually in $150-\mathrm{mm} \times 20$-mm plates (69 seedlings per plate) containing agar medium and grew M2 seedlings for $10 \mathrm{~d}$ under long-day conditions. We excised two leaves from each seedling and then carried out histochemical GUS assays in the 96-well plates. We then transferred putative mutants with GUS staining in the leaves to soil to set seeds (M3). We carried out GUS assays for 5-10 seedlings of M3 to isolate mutants that inherited GUS expression. We backcrossed acg1 mutants with wild-type plants and tested the resulting $\mathrm{F}_{1}$ seeds for both the late-flowering phenotype and GUS expression. We scored the $F_{2}$ populations obtained from self-pollinated $F_{1}$ plants for segregation with the late-flowering phenotype. We tested the late-flowering $F_{3}$ populations for GUS expression by the histochemical method. For genetic mapping of the acgl mutation, we crossed acgl with the ecotype Landsberg erecta. We selected homozygous acgl mutants in the segregated $\mathrm{F}_{2}$ population based on the late-flowering phenotype. We mapped the mutation using SSLP markers HYB7, HYB8 and PLS2. Primer sequences are available on request.

Analysis of GUS expression. We carried out histochemical assays of GUS activity by incubating the treated seedlings in 5-bromo-4-chloro-3-indolyl glucuronide (DUCHEFA) at $37^{\circ} \mathrm{C}$ for $24 \mathrm{~h}$ and removing the chlorophyll from green tissues by incubation in $100 \%$ ethanol, as described ${ }^{23}$. We carried out quantitative GUS assays in tissue extracts by fluorometric measurement of 4methylumbelliferone produced from the $\beta$-D-glucuronide precursor ${ }^{23}$.

Analysis of gene expression. We extracted total RNA using TRI Reagent (Molecular Research Center) and carried out northern-blot analysis as previously described ${ }^{9}$. We analyzed expression of the genes FLC, SOC1, FT and TUBB (encoding $\beta$-tubulin) by RT-PCR as described ${ }^{24}$. DNA probes used for northern-blot analysis and primer sequences and conditions for amplification are available on request.

Electrolyte leakage test. We determined freezing-induced electrolyte leakage from fully developed rosette leaves from 2-week-old plants grown in soil as described ${ }^{10}$. We placed one excised leaflet in a 5-ml test tube containing $100 \mu \mathrm{l}$ of deionized water in a refrigerated circulator bath (PolyScience) at $0{ }^{\circ} \mathrm{C}$ and programmed the temperature of the bath to decrease to $-9{ }^{\circ} \mathrm{C}$ in $1-{ }^{\circ} \mathrm{C}$ increments over $30 \mathrm{~min}$. We used at least 10 samples for each temperature.

Sequencing of DNA from mutants. We extracted genomic DNA of mutants using DNeasy plant mini-kit (Qiagen) and amplified the FVE genomic region by PCR using two sets of specific primers, HYB15F and MJH6-2, and HYB6 and HYB15R. Primer sequences are available on request. We sequenced six independent PCR products for each amplified DNA fragment.

Note: Supplementary information is available on the Nature Genetics website.

\section{ACKNOWLEDGMENTS}

We thank R. Amasino for $f l d-3$, fve-4, fve- $4 f c-3$ and $f l c-3$ seeds and for comments on the manuscript and J.M. Martínez-Zapater for sharing unpublished data. This work was supported by grants from Plant Diversity Research Center of 21st Century Frontier Research Program (J.K. and I.L.) funded by Ministry of Science and Technology of Korean Government and from Agricultural Plant Stress Research Center (J.K.) funded by Korea Science and Engineering Foundation.

\section{COMPETING INTERESTS STATEMENT}

The authors declare that they have no competing financial interests. 
Received 11 December 2003; accepted 14 January 2004

Published online at http://www.nature.com/naturegenetics/

1. Thomashow, M.F. Plant cold acclimation: freezing tolerance genes and regulatory mechanisms. Ann. Rev. Plant Physiol. Plant Mol. Biol. 50, 571-599 (1999).

2. Shinozaki, K. \& Yamaguchi-Shinozaki, K. Molecular responses to dehydration and low temperature: differences and cross-talk between two stress signaling pathways. Curr. Opin. Plant Biol. 3, 353-360 (2000).

3. Yamaguchi-Shinozaki, K. \& Shinozaki, K. A novel cis-acting element in an Arabidopsis gene is involved in responsiveness to drought, low temperature, or highsalt stress. Plant Cel/ 6, 251-264 (1994).

4. Baker, S.S., Wilhelm, K.S. \& Thomashow, M.F. The 5'-region of Arabidopsis thaliana cor15a has cis-acting elements that confer cold-, drought- and ABA-regulated gene expression. Plant Mol. Biol. 24, 701-713 (1994).

5. Jaglo, K.R. et al. Components of the Arabidopsis C-repeat/dehydration- responsive element binding factor cold-response pathway are conserved in Brassica napus and other plant species. Plant Physiol. 127, 910-917 (2001).

6. Michaels, S.D. \& Amasino, R.M. FLOWERING LOCUS $C$ encodes a novel MADS domain protein that acts as a repressor of flowering. Plant Cell 11, 949-956 (1999).

7. Brehm, A. et al. Retinoblastoma protein recruits histone deacetylase to repress transcription. Nature 391, 597-601 (1998).

8. Nicolas, E. et al. RbAp48 belongs to the histone deacetylase complex that associates with the retinoblastoma protein. J. Biol. Chem. 275, 9797-9804 (2000).

9. Kim, H.-J., Kim, Y.-K., Park, J.-Y. \& Kim, J. Light signalling mediated by phytochrome plays an important role in cold-induced gene expression through the C-repeat/dehydration responsive element (C/DRE) in Arabidopsis thaliana. Plant J. 29, 693-704 (2002).

10. Ishitani, M., Xiong, L., Lee, H., Stevenson, B. \& Zhu, J.-K. HOS1, a genetic locus involved in cold-responsive gene expression in Arabidopsis. Plant Cell 10, 1151-1161 (1998).

11. Simpson, G.G. \& Dean, C. Arabidopsis, the rosetta stone of flowering time? Science 296, 285-289 (2002).
12. Macknight, R. et al. FCA, a gene controlling flowering time in Arabidopsis, encodes a protein containing RNA-binding domains. Cel/ 89, 737-745 (1997).

13. Koornneef, M., Hanhart, C.J. \& Van deer Veen, J.H. A genetic and physiological analysis of late flowering mutants in Arabidopsis thaliana. Mol. Gen. Genet. 229, 57-66 (1991).

14. Putterill, J., Robson, F., Lee, K., Simon, R. \& Coupland, G. The CONSTANS gene of Arabidopsis promotes flowering and encodes a protein showing similarities to zinc finger transcription factors. Cell 80, 847-857 (1995).

15. Samach, A. et al. Distinct roles of CONSTANS target genes in reproductive development of Arabidopsis. Science 288, 1613-1616 (2000).

16. Sheldon, C.C. et al. The control of flowering by vernalization. Curr. Opin. Plant Biol. 3, 418-422 (2000).

17. Ausín, I., Alonso-Blanco, C., Jarillo, J.A., Ruiz-García, L. \& Martínez-Zapater, J.M. Regulation of flowering time by FVE, a retinoblastoma-associated protein. Nat. Genet. advance online publication, 25 January 2004 (doi:10.1038/ng1295).

18. Smith, T.F., Gaitatzes, C., Saxena, K. \& Neer, E.J. The WD repeat: a common architecture for diverse functions. Trends Biochem. Sci. 24, 181-185 (1999).

19. Rossi, V. et al. A maize histone deacetylase and retinoblastoma-related protein physically interact and cooperate in repressing gene transcription. Plant Mol. Biol. 51, 401-413 (2003)

20. Wu, K., Tian, L., Zhou, C., Brown, D. \& Miki, B. Repression of gene expression by Arabidopsis HD2 histone deacetylases. Plant J. 34, 241-247 (2003).

21. Blázquez, M.A., Ahn, J.H. \& Weigel, D. A thermosensory pathway controlling flowering time in Arabidopsis thaliana. Nat. Genet. 33, 168-171 (2003).

22. He, Y., Michaels, S.D. \& Amasino, R.M. Regulation of flowering time by histone acetylation in Arabidopsis. Science advance online publication, 30 October 2003 (doi:10.1126/science.1091109).

23. Jefferson, R.A. \& Wilson, K.J. The GUS gene fusion system. Plant Mol. Biol. Manual B14, 1-33 (1991).

24. Lee, $\mathrm{H}$. et al. The AGAMOUS-LIKE 20 MADS domain protein integrates floral inductive pathways in Arabidopsis. Genes Dev. 14, 2366-2376 (2000).

25. Falquet, L. et al. The PROSITE database, its status in 2002. Nucleic Acids Res. $\mathbf{3 0}$, 235-238 (2002) 